\title{
Integrative Analysis of Transcriptome and MicroRNome Profiles in Cholangiocarcinoma
}

Fábio E Severino ${ }^{1,2}$, Claudia N Hasimoto', Maria A M Rodrigues ${ }^{3}$, Juan C Llanos ${ }^{1}$ and Patricia P Reis ${ }^{1,2^{*}}$

${ }^{1}$ São Paulo State University (UNESP), Faculty of Medicine, Department of Surgery and Orthopedics, Botucatu, SP, Brazil

${ }^{2}$ São Paulo State University (UNESP), Faculty of Medicine, Experimental Research Unity (UNIPEX), Botucatu, SP, Brazil

${ }^{3}$ São Paulo State University (UNESP), Faculty of Medicine, Department of Pathology, Botucatu, SP, Brazil

\section{Abstract}

Aim: To identify deregulated expression of miRNAs and target genes in cholangiocarcinoma (CCA), as well as drug-gene interactions that may be useful for patient treatment.

Methods: We analyzed quantitative transcriptome and miRNA deep sequencing expression data from 45 samples, $36 \mathrm{CCA}$ and 9 histologically normal biliary tissues, obtained from the public repository The Cancer Genome Atlas (TCGA). Bioinformatic methods were used to identify and integrate differential expression of miRNA and transcriptome profiles in CCA vs. normal tissues. Deregulated miRNA and corresponding target genes were identified and mapped into miRNA-mRNA networks.

Results: Results showed 64 differentially expressed miRNAs (48 over and 16 under-expressed) between CCA and corresponding normal biliary tissues. Additionally, 432 genes (180 over and 252 under-expressed) were identified between CCA and normal samples. We identified individual miRNAs with the largest number of gene targets. Among these, miR-125a was over-expressed and had the highest number of direct interactions with 33 mRNA targets. miRNAs miR-122 and miR-139 were the under-expressed miRNAs with the highest number of interactions (9 targets each). miR-122 was found to modulate the expression of the transcription factor FOXM1, known to be involved in tumorigenesis and the matrix metalloproteinase MMP7, an important mediator of tumor invasion. miR-148 and miR-194 were predicted to modulate NQO1, which is up-regulated in cancer and associated with treatment resistance in cholangiocarcinoma.

Conclusion: The novelty of our study is the identification of complex deregulated networks of miRNAs and target genes in CCA. miRNAs with a large number of targets may have a higher functional impact on cell regulation. These findings contribute for a better understanding of CCA biology. Identified miRNAs and target genes are potential candidates for the design of validation strategies towards the characterization of clinically applicable biomarkers; such biomarkers may be useful for the development of molecularly-targeted therapeutics that can benefit patients.

Keywords: Cholangiocarcinoma; Transcriptome; MicroRNAs; Gene networks; Pathways

\section{Introduction}

Cholangiocarcinoma (CCA) is a malignant tumor of the liver that arises from epithelial cells of the bile ducts, both within $(\sim 10 \%)$ and outside $(\sim 90 \%)$ of the liver $[1,2]$. Although rare, it is an aggressive tumor associated with a poor prognosis. Survival rates around $15 \%$ at 2 years after diagnosis have been reported in the USA [2]. A recent epidemiology study in the USA showed that mortality rates by CCA have increased significantly in the past decade: for individuals $>25$ years old, there was a $36 \%$ increase in mortality between 1999-2014: 2.2 to 3 per 100,000, and deaths were the highest among African Americans (45\%), followed by Asians (22\%) and whites (20\%) [3]. In Brazil, 8,700 deaths by CCA combined with all liver cancers were reported in 2013 [4]. In Asia, the Japanese population has relatively higher rates of CCA, with an incidence of 3.5 and 3.05 cases per 100,000 in Osaka and Hiroshima, respectively. Other countries such as Singapore, Philippines and Vietnam have a lower incidence of $1.45,1.2$ and 0.1 respectively, per 100,000 people [5]. The incidence and mortality by intrahepatic CCA in Japan are similar to those other countries, however, incidence of extrahepatic CCA is higher [6].

Risk factors for development of CCA include chronic inflammation of the biliary tree, seen in primary sclerosing cholangitis, congenital fibropolycystic diseases of the biliary tract, HCV infection, previous exposure to Thorotrast, used in radiography of the biliary tree and infection of the biliary tracked by Clonorchis sinensis, common in southeast Asia [2]. Cholangiocarcinoma is often difficult to diagnose [7] and treat with conventional therapies due to the high intra and intertumoral genetic heterogeneity [8]. Surgical treatment, when possible, remains the best option to achieve better survival. Therefore, there is an urgent need for the development of genomic-driven treatment strategies for patients with this aggressive form of cancer.

MicroRNAs (miRNAs) are small RNAs of 18-22 nucleotides in length, non-protein coding and are potent gene expression regulators. A canonical mode of action of miRNAs is through binding to 3'-UTR of mRNA, leading to translational inhibition or mRNA degradation [9]. To date, 2,588 mature miRNA sequences have been identified in the human genome according to miRBase (http://www.mirbase.

*Corresponding author: Patricia P Reis, Ph. D, São Paulo State University (UNESP), Faculty of Medicine, Department of Surgery and Orthopedics. Botucatu, SP. CEP 18618-687, Brazil, Tel: 551438801635; E-mail: preis@fmb.unesp.br

Received June 22, 2017; Accepted August 04, 2017; Published August 10, 2017 Citation: Severino FE, Hasimoto CN, Rodrigues MAM, Llanos JC, Reis PP (2017) Integrative Analysis of Transcriptome and MicroRNome Profiles in Cholangiocarcinoma. Cancer Sci Ther 9: 580-588. doi: 10.4172/19485956.1000477

Copyright: (c) 2017 Guei-Fen C, et al. This is an open-access article distributed under the terms of the Creative Commons Attribution License, which permits unrestricted use, distribution, and reproduction in any medium, provided the original author and source are credited. 
org/cgi-bin/mirna_summary.pl?org=hsa) [10]. More recently, a deep sequencing analysis of 13 different cell types identified 3,707 novel mature miRNAs, suggesting that there may be several more miRNA sequences still unknown in the human genome [11].

MiRNAs regulate genes with functions in embryonic development, differentiation, apoptosis, cell proliferation [12-14] and genes involved in oncogenesis [15-17]. miRNAs and their target genes have been shown to be potentially useful to help improve diagnosis, prognosis and treatment of patients with cancer [18-23].

Expression and functional assessment of the role of miRNAs in CCA have been shown in previous studies. However, most studies, as reviewed in [24], have been mainly focused on candidate miRNAs rather than performing a large comprehensive miRNA expression analysis. Therefore, there is still a need to better understand the regulatory pathways involved in the pathogenesis of this aggressive cancer.

A recent study by Farshidfar et al. reported The Cancer Genome Atlas (TCGA) biomarker analysis of CCA through integrative analysis of copy number, gene expression, methylation and somatic mutations. This study identified distinct histological subtypes enriched for the presence of isocitrate dehydrogenase (IDH) mutations and compared global genomic changes occuring in cancers of the liver, pancreas and the biliary tree [25]. Our study used this same cholangiocarcinoma -TCGA dataset however it was focused on the role of global miRNA expression changes in transcriptome regulation. Our integrative analysis of transcriptome and miRNA expression data contributes to better understand a number of functionally relevant pathways in CCA. Such studies may lead to the identification of clinically applicable biomarkers to improve diagnosis, patient treatment and survival.

\section{Materials and Methods}

Global transcriptome and miRNA expression data were retrieved from 45 samples (36 CCA and 9 histologically normal biliary tissues) profiled by TCGA (http://cancergenome.nih.gov/). Raw data (level 3, read counts) were quantified by counting transcriptome sequencing (RNA-Seq) and miRNA sequencing (miRNA-Seq) reads. Quantitative data were used to calculate differential mRNA and miRNA expression levels in CCA relative to normal biliary tissues. R/Bioconductor EBSeq [26] and edgeR package [27] were used in their default settings, for mRNA (gene) and miRNA expression analyses and Multidimensional scaling (MDS) analysis, respectively. A list of differentially expressed (DE) genes and miRNAs was generated and fold change (FC) expression values were determined. Genes and miRNAs were identified as over- or under-expressed with FDR $\leq 0.05$. Differentially expressed genes with $\mathrm{FC} \geq 3$ and miRNAs with $\mathrm{FC} \geq 1$, based on their quartiles (Q3), were used in subsequent analyzes. The lists of differentially expressed genes and miRNAs in CCA compared to normal tissues were overlapped and miRNA target genes were identified. Identification of miRNA target genes was performed using microRNA Data Integration Portal (mirDIP) (http://ophid.utoronto.ca/mirDIP) [28]. External data validation was performed to compare differentially expressed miRNAs identified through analysis of TCGA data with the current literature (Supplementary Table 1). Furthermore, we searched DisGeNET, a database containing gene-disease associations (http://www.disgenet. org/web/DisGeNET/menu/home) [29] with the goal of enriching deregulated genes in CCA and that were also found deregulated in the TCGA dataset.

Next, in order to achieve a systematic understanding of deregulated networks associated with disease biology, miRNA target genes and their respective proteins were used to construct protein-protein interaction (PPI) networks. PPI networks were identified using STRING, a search tool for the retrieval of interacting Genes/Proteins (http://string-db. org/) [30]. The DGIdb database (http://dgidb.genome.wustl.edu/) [31] and STITCH (http://stitch.embl.de/) [32] were used to identify potential interactions between genes and proteins. Visualization and analysis of network properties of graphs such as Degree [33] were performed in Cytoscape [34,35].

\section{Results}

Results presented herein are in whole based on our analysis and interpretation of data generated by the TCGA research network (http:// cancergenome.nih.gov/). We identified 64 miRNAs differentially expressed between CCA and corresponding normal tissues from the TCGA dataset (Supplementary Table 2). Of these, 48 were over- and 16 were under-expressed, as described in Table 1. In addition, we identified 432 differentially expressed genes between CCA and normal tissues with FDR $<0.05$. Of the 432 genes, 180 were over- and 252 were underexpressed. Supplementary Table 3 lists all genes identified including this 432 gene subset. The top 20 over- and under-expressed genes are highlighted in Table 2.

To visualize the separation between the different classes of samples (normal and tumor), a MDS graph was elaborated with the data set of miRNAs available for cholangiocarcinoma (Figure 1). Among the 48 over-expressed miRNAs, miR-125a was identified to have the highest number of direct interactions (33 mRNA targets), followed by miR-27a (17 targets), miR-127 (15 targets) and miR-200a (14 targets) (Figure 2). A subset of genes was found to interact with a large number of miRNAs, such as the under-expressed genes $A C A D S B$, which interacts with 19 miRNAs, KMO with 17 miRNAs and INSIG1 interacting with

\begin{tabular}{|c|c|c|c|c|c|}
\hline \multicolumn{5}{|c|}{ Up } & \multicolumn{2}{c|}{ Down } \\
\hline miRNA & log2 FC & miRNA & log2 FC & miRNA & log2 FC \\
\hline let-7e & 1,56 & miR-218 & 1,91 & let-7c & $-1,89$ \\
\hline let-7i & 1,10 & miR-221 & 1,99 & miR-101 & $-1,83$ \\
\hline miR-10b & 2,58 & miR-222 & 2,16 & miR-122 & $-3,91$ \\
\hline miR-1180 & 1,33 & miR-224 & 1,50 & miR-139 & $-2,54$ \\
\hline miR-125a & 1,15 & miR-23a & 1,70 & miR-144 & $-1,98$ \\
\hline miR-127 & 1,04 & miR-24 & 1,01 & miR-148a & $-2,50$ \\
\hline miR-136 & 1,02 & miR-25 & 1,03 & miR-192 & $-1,73$ \\
\hline miR-141 & 4,88 & miR-27a & 2,15 & miR-194 & $-1,46$ \\
\hline miR-143 & 1,09 & miR-30a & 1,12 & miR-22 & $-1,08$ \\
\hline miR-150 & 1,17 & miR-30b & 1,26 & miR-378 & $-2,70$ \\
\hline miR-155 & 1,52 & miR-30d & 1,33 & miR-424 & $-1,42$ \\
\hline miR-16-2 & 1,18 & miR-338 & 1,35 & miR-451 & $-1,17$ \\
\hline miR-181a & 1,57 & miR-375 & 2,26 & miR-455 & $-1,53$ \\
\hline miR-181b & 2,31 & miR-381 & 1,16 & miR-505 & $-1,85$ \\
\hline miR-181c & 2,06 & miR-382 & 1,42 & miR-574 & $-1,16$ \\
\hline miR-182 & 4,69 & miR-409 & 1,32 & miR-99a & $-2,07$ \\
\hline miR-183 & 5,03 & miR-410 & 1,69 & -- & -- \\
\hline miR-200a & 2,84 & miR-429 & 2,94 & -- & -- \\
\hline miR-200b & 2,99 & miR-452 & 1,05 & -- & -- \\
\hline miR-200c & 5,29 & miR-598 & 1,59 & -- & -- \\
\hline miR-203 & 1,02 & miR-625 & 1,88 & -- & -- \\
\hline miR-21 & 2,72 & miR-9 & 1,75 & -- & -- \\
\hline miR-210 & 1,13 & miR-92b & 3,13 & -- & -- \\
\hline miR-215 & 1,30 & miR-99b & 1,48 & & \\
\hline Up: Up Regulated miRNAs; Down: Down-Regulated miRNAs; FC: Fold Change. \\
\hline
\end{tabular}

Table 1: Differentially expressed miRNAs identified in cholangiocarcinoma compared to normal biliary tissues. 
Citation: Severino FE, Hasimoto CN, Rodrigues MAM, Llanos JC, Reis PP (2017) Integrative Analysis of Transcriptome and MicroRNome Profiles in Cholangiocarcinoma. Cancer Sci Ther 9: 580-588. doi: 10.4172/1948-5956.1000477

15 miRNAs. Predicted interactions between under-expressed miRNAs and over-expressed genes in CCA are shown in Figure 3. Among the under-expressed miRNAs, miR-122 and miR-139 were found to have the highest number of interactions ( 9 targets each), followed by miR-424 and miR-194 with 8 targets each. miR-122 was predicted to target the transcription factor FOXM1, the matrix metalloproteinase $M M P 7$ and the TGFß regulator PMEPA1. Among these genes, FOXM1 and MMP7 were specifically deregulated in CCA cells. In addition, miR-148 and miR-194 were predicted to target $\mathrm{NAD}(\mathrm{P}) \mathrm{H}$ quinone dehydrogenase 1

\begin{tabular}{|c|c|c|c|}
\hline \multicolumn{2}{|c|}{ Up, top 20 genes } & \multicolumn{2}{c|}{ Down, top 20 genes } \\
\hline Gene & log2 FC & Gene & log2 FC \\
\hline AHNAK2 & 5,33 & A1BG & $-6,03$ \\
\hline BAIAP2L2 & 5,14 & ACSL1 & $-5,27$ \\
\hline C12orf75 & 5,03 & ALB & $-5,99$ \\
\hline C1orf106 & 5,17 & ALDOB & $-5,34$ \\
\hline EPCAM & 4,74 & APOC1 & $-5,49$ \\
\hline ITGA2 & 4,75 & CYP2D6 & $-5,76$ \\
\hline ITGB4 & 4,95 & CYP2E1 & $-6,20$ \\
\hline ITIH5 & 5,83 & DCXR & $-5,30$ \\
\hline KRT19 & 4,85 & GLS2 & $-5,25$ \\
\hline LAMC2 & 5,61 & HP & $-5,65$ \\
\hline MFI2 & 4,92 & HPX & $-5,81$ \\
\hline MMP11 & 5,76 & ITIH4 & $-5,55$ \\
\hline MMP7 & 4,85 & KMO & $-5,31$ \\
\hline NDRG4 & 4,92 & MST1 & $-5,18$ \\
\hline NQO1 & 5,14 & MT1G & $-6,17$ \\
\hline PPAP2C & 4,66 & MT1X & $-5,32$ \\
\hline SEZ6L2 & 5,25 & SARDH & $-5,39$ \\
\hline SPP1 & 6,18 & SDS & $-5,34$ \\
\hline TESC & 5,24 & SLC27A5 & $-6,41$ \\
\hline TMEM132A & 5,63 & SLC47A1 & $-5,18$ \\
\hline Up: Up Regulated Genes; Down: Down Regulated Genes; FC: Fold Change. \\
\hline
\end{tabular}

Table 2: Differentially expressed genes identified in cholangiocarcinoma compared to normal tissues.

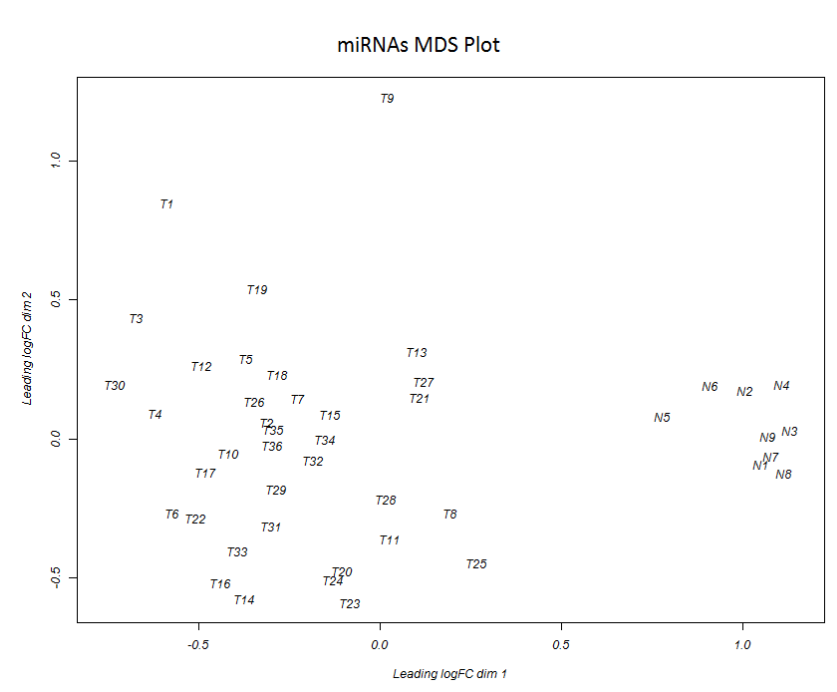

Figure 1: Multidimensional scaling (MDS) plot obtained using normalized counts per million (CPM) miRNA expression data in normal and cholangiocarcinoma tissues. In this plot, the distances correspond to leading log-fold-changes between each pair of samples. The leading log-fold-change is the average (root-mean-square) of the largest absolute log-fold-changes between each pair of samples. N: Normal and T: Tumor.
(NQO1) gene. The over-expressed genes PMEPA1 and ITGA2, ITGA3 and IGSF3 were found to be targeted by at least five miRNAs. PPI networks including enrichment analysis of biological functions were constructed for over- (Figure 4) and under-expressed genes (Figure 5). Furthermore, we identified potential drugs with validated drug-gene interaction data (Figure 6).

\section{Discussion and Conclusion}

Since miRNAs are potent gene expression regulators targeting multiple genes in several pathways, precise identification of miRNA expression in cancer compared to paired normal tissues can shed light into the mechanisms that determine cell fate and function related to disease development and progression.

Herein, deregulated expression of miRNAs was found to modulate a wide range of genes in disease-associated pathways. Among these, miR-365, which plays a role in chromatin modification, was downregulated in CCA compared to normal biliary tissues (Supplementary Table 2) and has also been described in other tumor types [36]. miR-365a also regulates genes related to epithelial to mesenchymal transition, cell cycle progression, and PI3K/AKT and MAPK pathways. Notably, a chromatin modifier gene expression signature was reported in cholangiocarcinoma tumor subtypes harboring IDH mutations [25].

Previous studies in other cancer types [37-40] have also provided supporting evidence for the involvement of genes and miRNAs identified as deregulated in CCA. Interestingly, miR-24 up-regulation was also detected in Acute Myeloid Leukemia [41] and in hepatocellular carcinoma being correlated with larger tumor size, higher microvessel density and tumor dedifferentiation, which are important factors associated with cancer cell invasion [42].

Among known miRNA clusters, miR-183 (miR-183, miR-96 and miR-182) was over-expressed in CCA compared to normal biliary tissues. miR-183 cluster has been reported as highly expressed in cancer

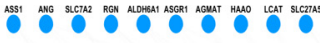 800060 0 0000

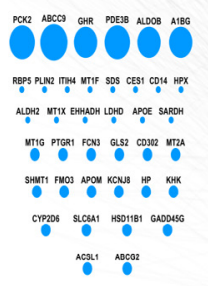

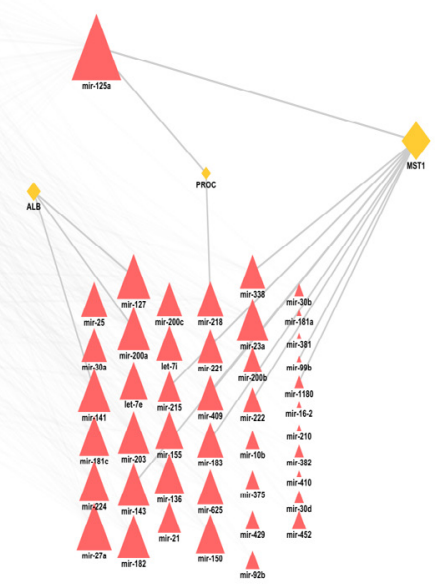

Figure 2: Interaction network between over-expressed miRNAs and underexpressed mRNA targets. Gene targets were predicted by mirDIP and found as differentially expressed in our analysis. miRNAs are shown in red and mRNA targets in blue. Genes deregulated in cholangiocarcinoma are highlighted as yellow diamonds (data obtained from DisGeNET database). Size of the circles and triangles is proportional to the number of direct interactions (Degree). 
Citation: Severino FE, Hasimoto CN, Rodrigues MAM, Llanos JC, Reis PP (2017) Integrative Analysis of Transcriptome and MicroRNome Profiles in Cholangiocarcinoma. Cancer Sci Ther 9: 580-588. doi: 10.4172/1948-5956.1000477

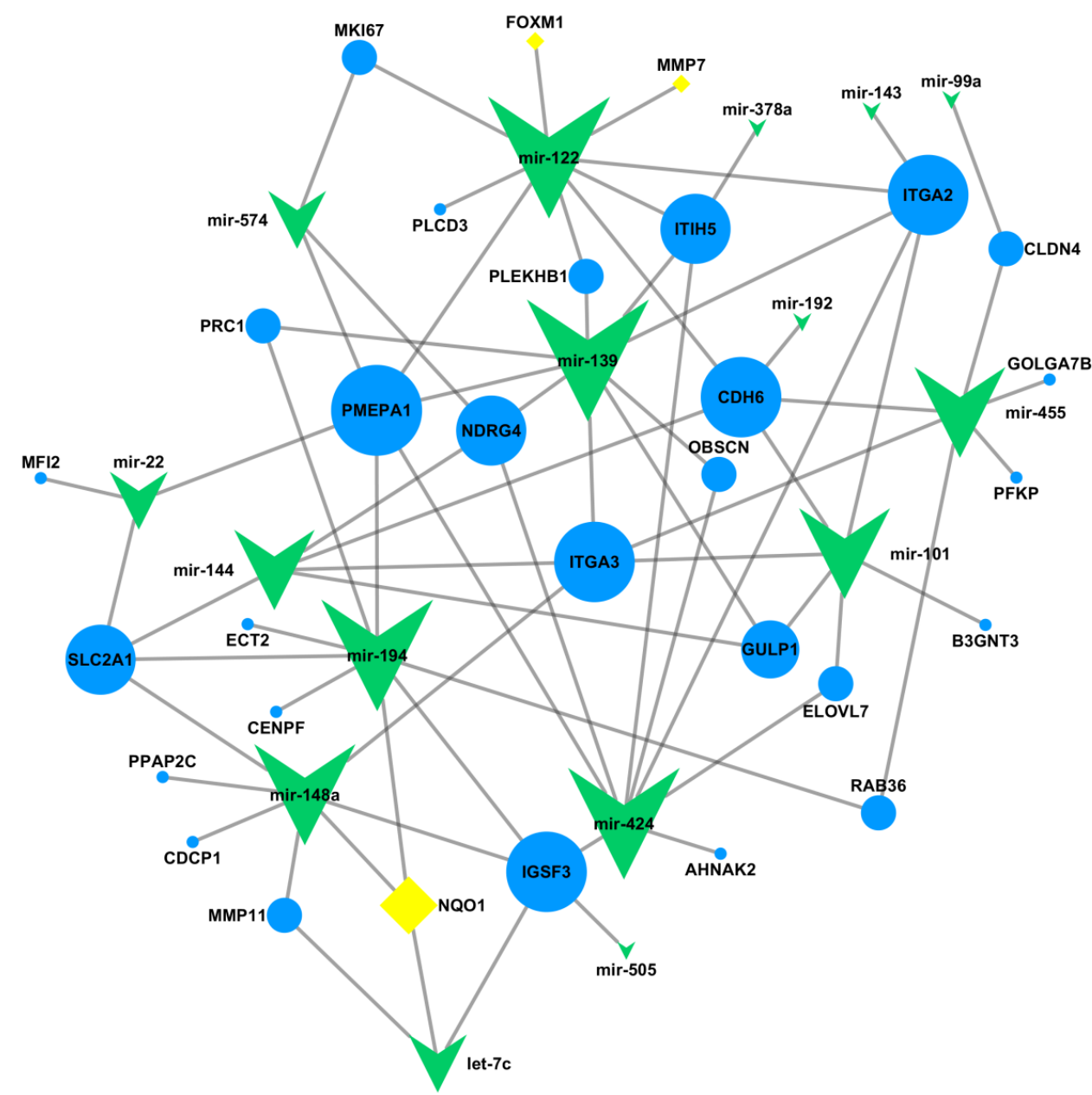

Figure 3: Interaction network between under-expressed miRNAs and over-expressed mRNA targets. miRNAs are shown in green, target genes in blue, and genes that are deregulated in cholangiocarcinoma are highlighted as yellow diamonds (DisGeNET database). Size of the circles and triangles is proportional to the number of direct interactions (Degree).

[43]. miR-182 alone was found to be up-regulated in hepatocellular carcinoma [44,45] and suggested to act as a metastasis-inducing miRNA in the liver [46]. miR-182 may be a good candidate for functional validation studies as a therapeutic target in CCA.

miR-200 family was also over-expressed in CCA; this miRNA family consists of five members distributed in two chromosomes: cluster I on chromosome 1 (miR-200a, miR-200b, and miR-429) and cluster II on chromosome 12 (miR-200c and miR-141) [47]. Some members of this cluster, namely, miR-200a, miR-200b and miR-200c have increased expression in CCA [48]. miRNAs miR-21, miR-141 and miR-200b were reported as highly expressed in CCA and suggested to have a role in chemotherapy, since inhibition of miR-21 and miR-200b resulted in increased sensitivity of tumor cells to gemcitabine and inhibition of miR-141 led to decreased tumor cell growth [49]. miR-200 family has been also reported to be up-regulated in bladder carcinoma [50] and both miRNA clusters, miR-183 and miR-200 are positively regulated in ovarian cancer [51]. These miRNAs were suggested as potentially useful biomarkers for diagnosis and treatment of lung cancer [52].

The miR-148/152 cluster (miR-148a, miR-148b and miR-152) has been reported as commonly down-regulated in cancer [53], including
CCA [54]. Down-regulated expression of miR-148a and miR-152 were reported in breast [55] and gastric cancer [56,57], and under expression of miR-148a was reported in pancreatic cancer [58,59] and colorectal carcinoma [60]. Of note, in vivo replacement of miR-148a in hepatocellular carcinoma cells was shown to suppress and prevent tumor growth and reduce liver fibrosis [61]. Interestingly, a predicted target of the under-expressed miR-148, NQO1 gene, was found to have a large number of interactions and to be up-regulated in CCA. NQO1 over-expression occurs in different tumors compared to their normal surrounding tissues [62] and was recently associated with poor prognosis in serous ovarian cancer [63]. Cholangiocarcinoma is highly resistant to chemotherapy and $\mathrm{NQO1}$ protein, $\mathrm{NAD}(\mathrm{P}) \mathrm{H}$-quinone oxidoreductase 1 , has been shown to play a cooperative role in cancer chemoresistance [64]. Recent data by Zeekpudsa et al. [65] showed that NQO1 knock-down increased the response of CCA cells to the chemotherapeutic drugs 5 -fluorouracil and gemcitabine. Altogether, these findings suggest a potential role for NQO1 in treatment of patients with CCA.

Under-expression of miR-101 has been identified herein as well as in previous studies in liver cancer [66,67] and CCA [68]. Down- 


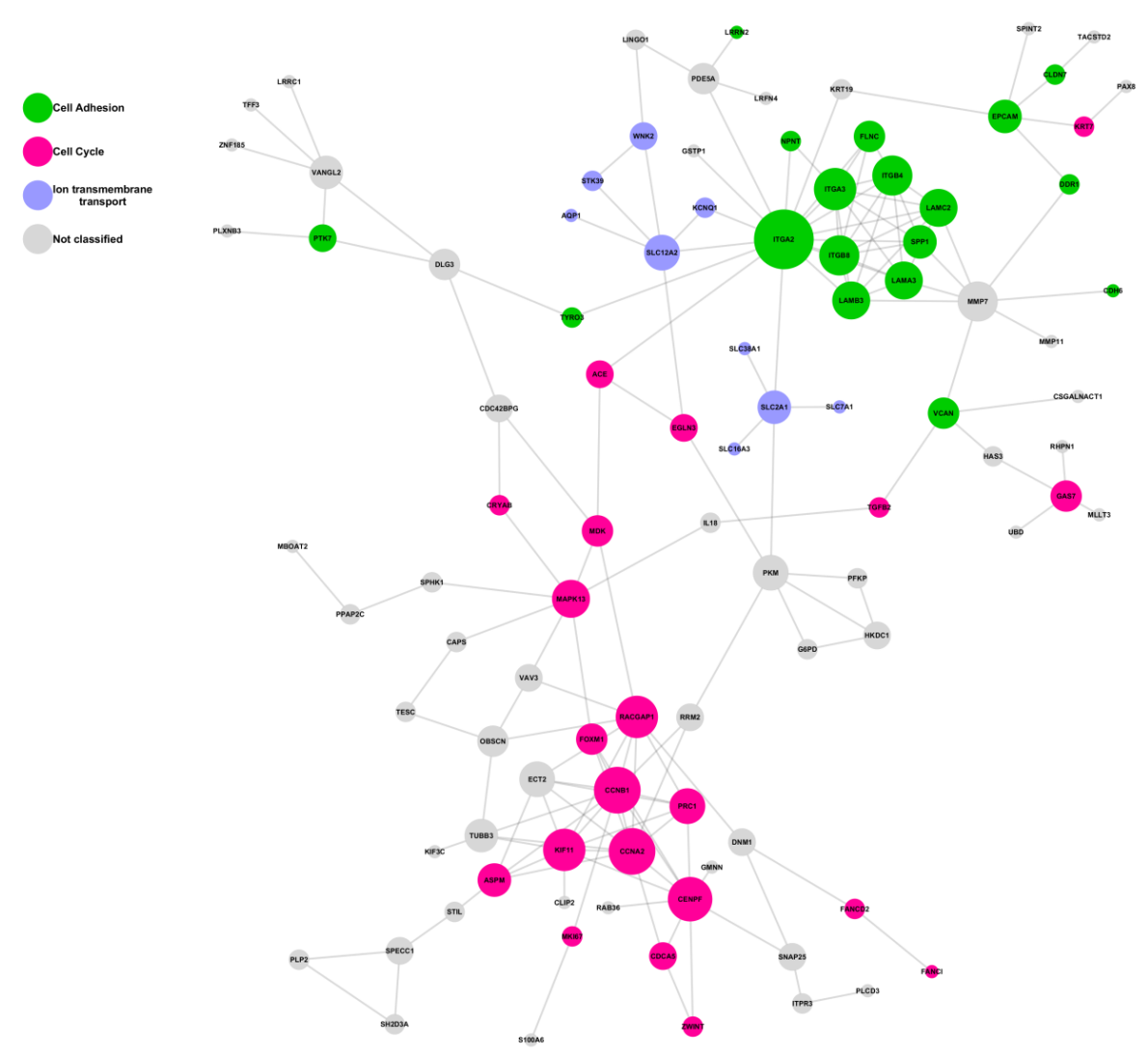

Figure 4: Protein-protein interaction network and Gene Ontology for biological function of deregulated genes in cholangiocarcinoma. Size of the circles is proportional to the betweenness centrality criteria. Bingo was used in its default settings with $p<0.05$ to detect biological functions.

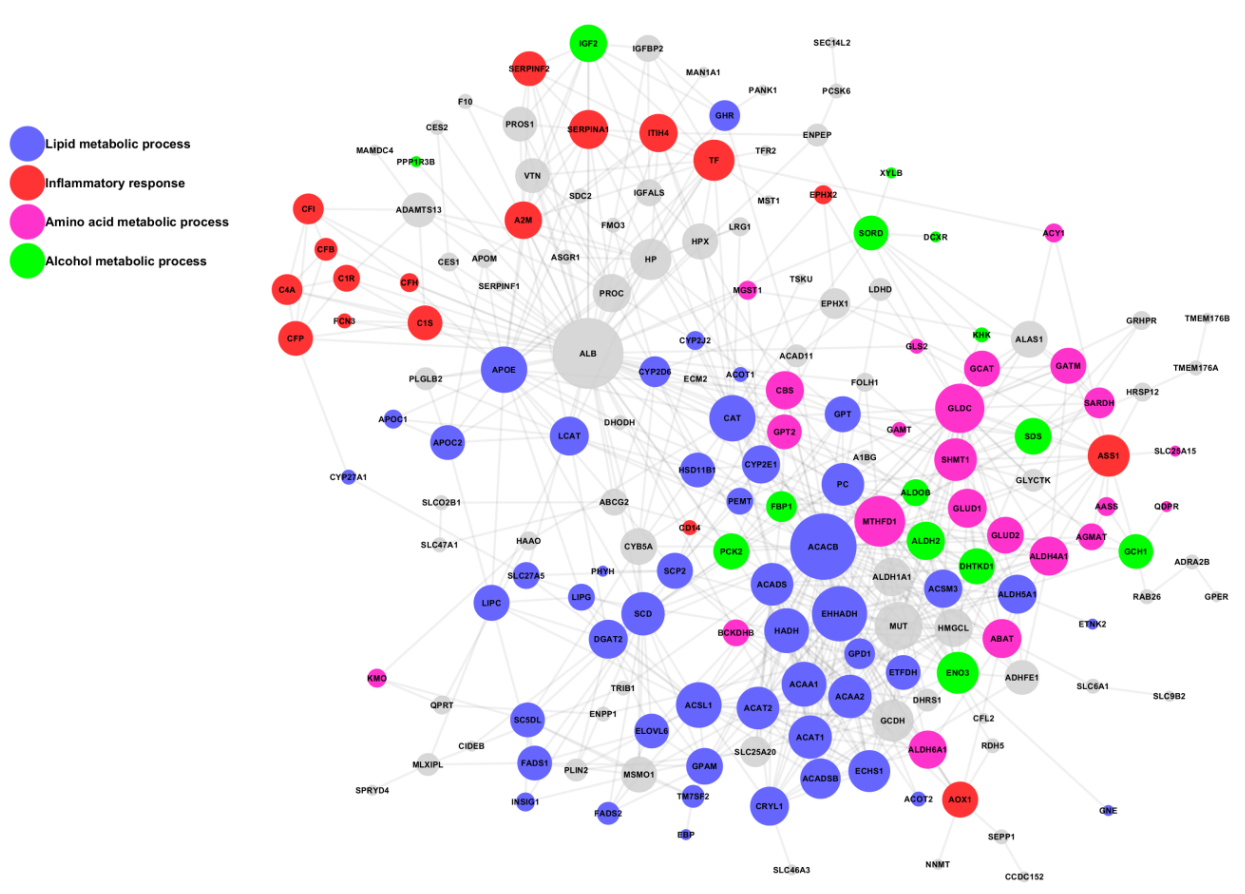

Figure 5: Protein-protein interaction network and Gene Ontology for biological function of deregulated genes in cholangiocarcinoma. Size of the circles is proportional to the betweenness centrality criteria. Bingo was used in its default settings with $p<0.05$ to detect biological functions. 

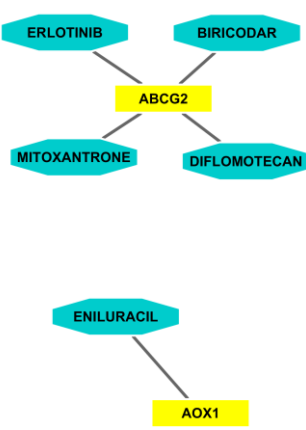
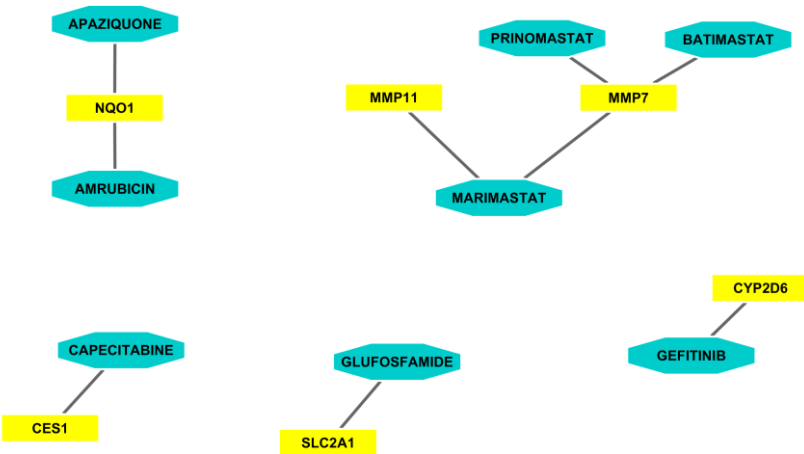

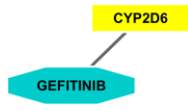

SLC2A1

Figure 6: Relationship between deregulated genes and chemotherapeutic drugs based on the DGIdb and STITCH database. Octagons represent currently available drugs and rectangles represent genes.

regulated miR-101 was associated with hepatitis B virus infection [69]. In CCA, miR-101 may modulate vascular endothelial growth factor (VEGF) and cyclooxygenase-2 (COX-2) expression levels leading to a decrease in tumor cell growth [68]. miR-101 is a putative regulator of genes linked to cell adhesion, including ITGA2, which has increased expression in CCA [70], as well as ITGA3 and CDH6.

According to our analyses, miR-125a was the over-expressed miRNA with the highest number of regulated target genes. Up-regulated miR-125a was also reported in prostate cancer [71]. However, other studies have reported under-expression of miR-125a and suggested a tumor suppressive role for this miRNA [72-75]. miR-125a can regulate the transcription of $A L B, P R O C$ and MST1, which are deregulated in CCA according to DisGeNET data. Interestingly, MST1 inactivation in mammalian liver has been demonstrated to increase hepatocyte proliferation and hepatomegaly followed by the development of hepatocellular carcinoma and CCA [76]. Among the under-expressed miRNAs, miR-122 targets the highest number of genes. miR-122 is down-regulated in hepatocellular carcinoma [77] and has a role in tumor development associated with the hepatitis $\mathrm{C}$ virus [78], since this miRNA is needed for viral replication [79]. miR-122 has been also shown to negatively regulate FOXM1, which has increased expression in CCA [70] and has been demonstrated to promote epithelial to mesenchymal transition and metastasis in hepatocellular carcinoma [80]. Inhibition of FOXM1 was suggested to have a role in cancer treatment [81] and miR-122 has been indicated as a potential target for replacement therapy in hepatocellular carcinoma [82].

Among the 180 genes found to be over-expressed in our analysis of this TCGA dataset, SPP1 (Osteopontin) was a hallmark. SPP1 overexpression has been reported in intrahepatic CCA [83]. SPP1 is involved in tumor cell adhesion, migration and invasion [84] and its serum levels were suggested to have prognostic value in hepatocellular carcinoma [85]. SPP1 over-expression has also been found in lung cancer [86], oral squamous cell carcinoma [87], colorectal [88], gastric and liver cancers [89]. SPP1 protein was associated with metastasis [90], demonstrated to interact with integrins [91] and matrix metalloproteinases (MMPs) in cancer [92].

MMPs are known extracellular matrix remodeling proteins with key roles in tumor cell migration, invasion, apoptosis, angiogenesis and immune cell migration [93]. MMPs have been suggested as prognostic biomarkers in CCA [94]. MMP11 is potentially regulated by the miRNAs let-7c and miR-148a, both with reduced expression levels as identified in our data analysis. MMP11 promotes cellular proliferation, angiogenesis and decrease cancer cell sensitivity to NK cells [95]. MMP11 is highly expressed in gastric tumors and siRNA inactivation of MMP11 led to decreased cancer growth [96]. Motrescu and Rio [97] showed that MMP11 is expressed in the tumor microenvironment in the adipose tissue around the tumor, acting in adipocyte dedifferentiation and leading to accumulation of non-malignant peritumoral fibroblastlike cells, which support tumor cell survival. In normal liver, MMP11 demonstrates constitutive expression [98] having IGF-BP1 (insulin-like growth factor binding protein 1 ) as a substrate and thus cooperating to increase the availability of IGF-1 [99]. Interestingly, MMP11 was the most significantly up-regulated gene in a CCA microarray study [100].

Our transcriptome analyses also identified MMP7 (Matrilysin) overexpression. One $M M P 7$ substrate is E-cadherin, an adherens junction protein involved in cell to cell interaction [101]. MMP7 has roles in tumorigenesis by cooperating with cellular growth and proliferation, having anti-apoptotic effects and inducing epithelial to mesenchymal transition and cell migration (reviewed in Gialeli) [95]. Abnormal $M M P 7$ protein levels were detected in CCA [99]. MMP7 is potentially regulated by miR-122, which was broadly under-expressed and targeting a large number of genes as identified herein. $M M P 7$ has been suggested as an useful biomarker in lung cancer [101] and CCA [98]. MMPs may be targeted by synthetic inhibitors such as Marimastat, a broad spectrum MMP inhibitor [95] that was experimentally tested and shown useful for treatment of polycystic liver disease, which presents cholangiocyte overgrowth by MMP hyperactivity [102]. In addition, Batimastat and Prinomastat are synthetic MMP inhibitors and Neovastat is a natural $M M P 7$ inhibitor [95]. Despite this and other published studies, there is a need to understand, at the protein level, the effects of miRNA-regulated and disease-associated pathways. Nevertheless, our study contributes for a better understanding of miRNA-modulated signaling pathways involved in CCA pathobiology. Future in vitro and in vivo functional validation studies targeting miRNAs and pathways are required for the development of novel treatment strategies for patients.

\section{Acknowledgements}

We acknowledge the contribution of the specimen donors and data generated by the TCGA research groups.

\section{Conflict of Interest}

The authors have no conflicts of interest to declare.

\section{References}

1. Govindan R, DeVita VT (2008) DeVita, Hellman, and Rosenberg's Cancer 
Citation: Severino FE, Hasimoto CN, Rodrigues MAM, Llanos JC, Reis PP (2017) Integrative Analysis of Transcriptome and MicroRNome Profiles in Cholangiocarcinoma. Cancer Sci Ther 9: 580-588. doi: 10.4172/1948-5956.1000477

Principles and Practice of Oncology Review. Lippincott Williams \& Wilkins, USA.

2. Kumar V, Abbas AK, Fausto N, Aster JC (2014) Robbins and Cotran Pathologic Basis of Disease, Professional Edition E-Book. Elsevier Health Sciences, Netherlands.

3. Yao KJ, Jabbour S, Parekh N, Lin Y, Moss RA (2016) Increasing mortality in the United States from cholangiocarcinoma: An analysis of the National Center for Health Statistics Database. BMC Gastroenterol 16: 117.

4. INCA (Incidence of Cancer in Brazil). Estimativa 2012-Incidência de Câncer no Brasil. 2012, 2016

5. Banales JM, Cardinale V, Carpino G, Marzioni M, Andersen JB, et al. (2016) Expert consensus document: Cholangiocarcinoma: Current knowledge and future perspectives consensus statement from the European Network for the Study of Cholangiocarcinoma (ENS-CCA). Nature reviews. Gastroenterol Hepatol 13: 261.

6. Utada M, Ohno Y, Tamaki T, Sobue T, Endo G (2014) Long-term trends in incidence and mortality of intrahepatic and extrahepatic bile duct cancer in Japan. J Epidemiol 24: 193-199.

7. Patel T (2011) Cholangiocarcinoma-controversies and challenges. Nature Reviews Gastroenterol Hepatol 8: 189-200.

8. Razumilava N, Gores GJ (2014) Cholangiocarcinoma. Lancet 383: 2168-2179.

9. Di Leva G, Calin GA, Croce CM (2006) MicroRNAs: Fundamental facts and involvement in human diseases. Birth Defects Research Part C: Embryo Today: Reviews 78: 180-189.

10. Kozomara A, Griffiths-Jones S (2013) miRBase: Annotating high confidence microRNAs using deep sequencing data. Nucleic Acids Res 42: D68-D73.

11. Londin E, Loher P, Telonis AG, Quann K, Clark P, et al. (2015) Analysis of 13 cell types reveals evidence for the expression of numerous novel primate-and tissue-specific microRNAs. Proc Natl Acad Sci 112: E1106-E1115.

12. Bartel DP (2004) MicroRNAs: Genomics, biogenesis, mechanism, and function. Cell 116: 281-297.

13. Harfe BD (2005) MicroRNAs in vertebrate development. Curr Opin Genet Dev 15: $410-415$

14. Cummins JM, Velculescu VE (2006) Implications of micro-RNA profiling for cancer diagnosis. Oncogene 25: 6220 .

15. Rinaldi A, Poretti G, Kwee I, Zucca E, Catapano CV, et al. (2007) Concomitant MYC and microRNA cluster miR-17-92 (C13orf25) amplification in human mantle cell lymphoma. Leuk Lymphoma 48: 410-412.

16. Frankel LB, Christoffersen NR, Jacobsen A, Lindow M, Krogh A, et al. (2008) Programmed cell death 4 (PDCD4) is an important functional target of the microRNA miR-21 in breast cancer cells. J Biol Chem 283: 1026-1033.

17. Reis PP, Tomenson M, Cervigne NK, Machado J, Jurisica I, et al. (2010) Programmed cell death 4 loss increases tumor cell invasion and is regulated by miR-21 in oral squamous cell carcinoma. Mol Cancer 9: 238.

18. Takamizawa J, Konishi H, Yanagisawa K, Tomida S, Osada H, et al. (2004) Reduced expression of the let-7 microRNAs in human lung cancers in association with shortened postoperative survival. Cancer Res 64: 3753-3756.

19. Lu J, Getz G, Miska EA, Alvarez-Saavedra E, Lamb J, et al. (2005) MicroRNA expression profiles classify human cancers Nature 435: 834-838.

20. Calin GA, Ferracin M, Cimmino A, Di Leva G, Shimizu M (2005) A microRNA signature associated with prognosis and progression in chronic lymphocytic leukemia. N Engl J Med 353: 1793-1801.

21. Yu SL, Chen HY, Chang GC, Chen CY, Chen HW (2008) MicroRNA signature predicts survival and relapse in lung cancer. Cancer cell 13: 48-57.

22. Yan LX, Huang XF, Shao Q, Huang MY, Deng L, et al. (2008) MicroRNA miR21 overexpression in human breast cancer is associated with advanced clinical stage, lymph node metastasis and patient poor prognosis. Rna 14: 2348-2360.

23. Iorio MV, Croce CM (2009) MicroRNAs in cancer: Small molecules with a huge impact. J Clin Oncol 27: 5848-5856.

24. Samuel N, Hudson TJ (2012) The molecular and cellular heterogeneity of pancreatic ductal adenocarcinoma. Nat Rev Gastroenterol Hepatol 9: 77-87.

25. Farshidfar F, Zheng S, Gingras MC, Newton Y, Shih J (2017) Integrative genomic analysis of cholangiocarcinoma identifies distinct IDH-mutant molecular profiles. Cell Rep 18: 2780-2794.

26. Leng N, Dawson JA, Thomson JA, Ruotti V, Rissman Al, et al. (2013) EBSeq An empirical Bayes hierarchical model for inference in RNA-seq experiments. Bioinformatics 29: 1035-1043.

27. Robinson MD, McCarthy DJ, Smyth GK (2010) edgeR: A bioconductor package for differential expression analysis of digital gene expression data. Bioinformatics 26: 139-140.

28. Shirdel EA, Xie W, Mak TW, Jurisica I (2011) NAViGaTing the micronomeusing multiple microRNA prediction databases to identify signalling pathwayassociated microRNAs. PloS one 6: e17429.

29. Piñero J, Queralt-Rosinach N, Bravo À, Deu-Pons J, Bauer-Mehren A, et al (2015) DisGeNET: A discovery platform for the dynamical exploration of human diseases and their genes. Database 2015.

30. Szklarczyk D, Franceschini A, Wyder S, Forslund K, Heller D, et al. (2014) STRING v10: Protein-protein interaction networks, integrated over the tree of life. Nucleic Acids Res 43: D447-D452.

31. Griffith M, Griffith OL, Coffman AC, Weible JV, McMichael JF, et al. (2013) DGIdb: Mining the druggable genome. Nature ethods 10: 1209-1210.

32. Kuhn M, Szklarczyk D, Pletscher-Frankild S, Blicher TH, Von Mering C, et al. (2013) STITCH 4: integration of protein-chemical interactions with user data. Nucleic Acids Res 42: D401-D407.

33. Pavlopoulos GA, Secrier M, Moschopoulos CN, Soldatos TG, Kossida S, et al (2011) Using graph theory to analyze biological networks. BioData Min 4: 10.

34. Shannon P, Markiel A, Ozier O, Baliga NS, Wang JT, et al. (2003) Cytoscape: A software environment for integrated models of biomolecular interaction networks. Genome Res 13: 2498-2504.

35. Cline MS, Smoot M, Cerami E, Kuchinsky A, Landys N, et al. (2007) Integration of biological networks and gene expression data using Cytoscape. Nat Protoc 2: 2366.

36. Moss TJ, Luo Z, Seviour EG, Sehgal V, Lu Y, et al. (2015) Genome-wide perturbations by miRNAs map onto functional cellular pathways, identifying regulators of chromatin modifiers. NPJ Syst Biol Appl 1: 15001.

37. Razumilava N, Bronk SF, Smoot RL, Fingas CD, Werneburg NW, et al. (2012 miR25 targets TNF-related apoptosis inducing ligand (TRAIL) death receptor4 and promotes apoptosis resistance in cholangiocarcinoma. Hepatology 55 $465-475$.

38. Collins AL, Wojcik S, Liu J, Frankel WL, Alder H, et al. (2014) A differential microRNA profile distinguishes cholangiocarcinoma from pancreatic adenocarcinoma. Ann Surg Oncol 21: 133-138.

39. Letelier P, García P, Leal P, Álvarez H, lli C et al. (2014) miR-1 and miR-145 act as tumor suppressor microRNAs in gallbladder cancer. Int J Clin Exp Patho 7: 1849.

40. Lin KY, Ye H, Han BW, Wang WT, Wei PP, et al. (2016) Genome-wide screen identified let-7c/miR-99a/miR-125b regulating tumor progression and stem-like properties in cholangiocarcinoma. Oncogene 35: 3376.

41. Organista-Nava J, Gómez-Gómez Y, Illades-Aguiar B, Del Carmen AlarcónRomero L, Saavedra-Herrera MV, et al. (2015) High miR-24 expression is associated with risk of relapse and poor survival in acute leukemia. Oncol Rep 33: 1639-1649.

42. Liu YX, Long XD, Xi ZF, Ma Y, Huang XY, et al. (2014) MicroRNA-24 modulates aflatoxin B1-related hepatocellular carcinoma prognosis and tumorigenesis. BioMed Res Int 2014.

43. Dambal S, Shah M, Mihelich B, Nonn L (2015) The microRNA-183 cluster: The family that plays together stays together. Nucleic Acids Res 43: 7173-7188.

44. Wang C, Ren R, Hu H, Tan C, Han M, et al. (2014) MiR-182 is up-regulated and targeting Cebpa in hepatocellular carcinoma. Chin J Cancer Res 26: 17.

45. Du C, Weng X, Hu W, Lv Z, Xiao H, et al. (2015) Hypoxia-inducible MiR-182 promotes angiogenesis by targeting RASA 1 in hepatocellular carcinoma. J Exp Clin Cancer Res 34: 67.

46. Wang J, Li J, Shen J, Wang C, Yang L, et al. (2012) MicroRNA-182 downregulates metastasis suppressor 1 and contributes to metastasis of hepatocellular carcinoma. BMC cancer 12: 227. 
Citation: Severino FE, Hasimoto CN, Rodrigues MAM, Llanos JC, Reis PP (2017) Integrative Analysis of Transcriptome and MicroRNome Profiles in Cholangiocarcinoma. Cancer Sci Ther 9: 580-588. doi: 10.4172/1948-5956.1000477

47. Humphries B, Yang C (2015) The microRNA-200 family: Small molecules with novel roles in cancer development, progression and therapy. Oncotarget 6: 6472.

48. Plieskatt J, Rinaldi G, Feng Y, Peng J, Easley S, et al. (2015) A microRNA profile associated with Opisthorchis viverrini-induced cholangiocarcinoma in tissue and plasma. BMC cancer 15: 309

49. Meng F, Henson R, Lang M, Wehbe $H$, Maheshwari S, et al. (2006) Involvement of human micro-RNA in growth and response to chemotherapy in human cholangiocarcinoma cell lines. Gastroenterology 130: 2113-2129.

50. Martínez-Fernández M, Dueñas M, Feber A, Segovia C, García-Escudero R, et al. (2015) A Polycomb-mir200 loop regulates clinical outcome in bladder cancer. Oncotarget 6: 42258 .

51. Wang L, Zhu MJ, Ren AM, Wu HF, Han WM, et al. (2014) A ten-microRNA signature identified from a genome-wide microRNA expression profiling in human epithelial ovarian cancer. PLoS One 9: e96472.

52. Lin Q, Mao W, Shu Y, Lin F, Liu S, et al. (2012) A cluster of specified microRNAs in peripheral blood as biomarkers for metastatic non-small-cell lung cancer by stem-loop RT-PCR. J Cancer Res Clin Oncol 138: 85-93.

53. Chen Y, Song YX, Wang ZN (2013) The microRNA-148/152 family: Multifaceted players. Mol Cancer 12: 43.

54. Braconi C, Huang N, Patel T (2010) MicroRNA-dependent regulation of DNA methyltransferase-1 and tumor suppressor gene expression by interleukin- 6 in human malignant cholangiocytes. Hepatology 51: 881-890.

55. Xu Q, Jiang Y, Yin Y, Li Q, He J, et al. (2012) A regulatory circuit of miR-148a/152 and DNMT1 in modulating cell transformation and tumor angiogenesis through IGF-IR and IRS1. J Mol Cell Biol 5: 3-13.

56. Chen Y, Song Y, Wang Z, Yue Z, Xu H, et al. (2010) Altered expression of MiR-148a and MiR-152 in gastrointestinal cancers and its clinical significance. J Gastrointest Surg 14: 1170-1179.

57. Sakamoto N, Naito Y, Oue N, Sentani K, Uraoka N, et al. (2014) MicroRNA-148a is downregulated in gastric cancer, targets MMP7, and indicates tumor invasiveness and poor prognosis. Cancer science 105: 236-243.

58. Hanoun N, Delpu Y, Suriawinata AA, Bournet B, Bureau C, et al. (2010) The silencing of microRNA 148a production by DNA hypermethylation is an early event in pancreatic carcinogenesis. Clinical chemistry 56: 1107-1118.

59. Munding JB, Vogt M, Kuhlmann JD, Verdoodt B, Nambiar S, et al (2011) MicroRNA-148a is down-regulated in human pancreatic ductal adenocarcinomas and regulates cell survival by targeting CDC25B. Lab Invest 91: 1472.

60. Takahashi M, Cuatrecasas M, Balaguer F, Hur K, Toiyama Y, et al. (2012) The clinical significance of MiR-148a as a predictive biomarker in patients with advanced colorectal cancer. PloS one 7: e46684.

61. Jung KH, Zhang J, Zhou C, Shen H, Gagea M, et al. (2016) Differentiation therapy for hepatocellular carcinoma: Multifaceted effects of miR-148a on tumor growth and phenotype and liver fibrosis. Hepatology 63: 864-879.

62. Danson S, Ward TH, Butler J, Ranson M (2004) DT-diaphorase: A target for new anticancer drugs. Cancer Treat Rev 30: 437-449.

63. Cui X, Li L, Yan G, Meng K, Lin Z, et al. (2015) High expression of NQO1 is associated with poor prognosis in serous ovarian carcinoma. BMC Cancer 15: 244

64. Tome ME, Frye JB, Coyle DL, Jacobson EL, Samulitis BK, et al. (2012) Lymphoma cells with increased anti-oxidant defenses acquire chemoresistance. Exp Ther Med 3: 845-852.

65. Zeekpudsa P, Kukongviriyapan V, Senggunprai L, Sripa B, Prawan A (2014) Suppression of $\mathrm{NAD}(\mathrm{P}) \mathrm{H}$-quinone oxidoreductase 1 enhanced the susceptibility of cholangiocarcinoma cells to chemotherapeutic agents. J Exp Clin Cancer Res 33: 11.

66. Zheng F, Liao YJ, Cai MY, Liu TH, Chen SP, et al. (2015) Systemic delivery of microRNA-101 potently inhibits hepatocellular carcinoma in vivo by repressing multiple targets. PLoS Genet 11: e1004873.

67. Cao K, Li J, Zhao Y, Wang Q, Zeng Q, et al. (2016) miR-101 inhibiting cell proliferation, migration and invasion in hepatocellular carcinoma through downregulating girdin. Mol Cells 39: 96-102

68. Zhang J, Han C, Zhu H, Song K, Wu T (2013) miR-101 inhibits cholangiocarcinoma angiogenesis through targeting vascular endothelial growth factor (VEGF). Am J Pathol 182: 1629-1639.

69. Xie Y, Yao Q, Butt AM, Guo J, Tian Z, et al. (2014) Expression profiling of serum microRNA-101 in HBV-associated chronic hepatitis, liver cirrhosis, and hepatocellular carcinoma. Cancer Biol Ther 15: 1248-1255.

70. Jinawath N, Chamgramol Y, Furukawa Y, Obama K, Tsunoda T, et al. (2006) Comparison of gene expression profiles between Opisthorchis viverrini and non-Opisthorchis viverrini associated human intrahepatic cholangiocarcinoma. Hepatology 44: 1025-1038.

71. Fu Y, Cao F (2015) MicroRNA-125a-5p regulates cancer cell proliferation and migration through NAIF1 in prostate carcinoma. Onco Targets Ther 8: $3827-$ 3835.

72. Guo X, Wu Y, Hartley RS (2009) MicroRNA-125a represses cell growth by targeting HuR in breast cancer. RNA Biol 6: 575-583.

73. Bi Q, Tang S, Xia L, Du R, Fan R, et al. (2012) Ectopic expression of MiR$125 a$ inhibits the proliferation and metastasis of hepatocellular carcinoma by targeting MMP11 and VEGF. PLoS One 7: e40169.

74. Tiwari A, Shivananda S, Gopinath KS, Kumar A (2014) MicroRNA-125a reduces proliferation and invasion of oral squamous cell carcinoma cells by targeting estrogen-related receptor $\alpha$ : Implications for cancer therapeutics. $J$ Biol Chem 289: 32276-32290.

75. Fan Z, Cui H, Xu X, Lin Z, Zhang X, et al. (2015) MiR-125a suppresses tumo growth, invasion and metastasis in cervical cancer by targeting STAT3. Oncotarget 6: 25266-25280.

76. Qin F, Tian J, Zhou D, Chen L (2013) Mst1 and Mst2 kinases: Regulations and diseases. Cell Biosci 3: 31.

77. Burchard J, Zhang C, Liu AM, Poon RT, Lee NP, et al. (2010) microRNA-122 as a regulator of mitochondrial metabolic gene network in hepatocellular carcinoma. Mol Syst Biol 6: 402.

78. Lau G, Marcellin P, Peters M (2007) Chronic hepatitis B: A global health problem requiring coherent worldwide treatment strategies. Hepatol Int 1 : 316-325.

79. Luna JM, Scheel TK, Danino T, Shaw KS, Mele A, et al. (2015) Hepatitis C virus RNA functionally sequesters miR-122. Cell 160: 1099-1110.

80. Meng FD, Wei JC, Qu K, Wang ZX, Wu QF, et al. (2015) FoxM1 overexpression promotes epithelial-mesenchymal transition and metastasis of hepatocellular carcinoma. World J Gastroenterol 21: 196-213.

81. Chan-On W, Huyen NT, Songtawee N, Suwanjang W, Prachayasittikul S, et al. (2015) Quinoline-based clioquinol and nitroxoline exhibit anticancer activity inducing FoxM1 inhibition in cholangiocarcinoma cells. Drug Des Devel Ther 9: 2033-2047.

82. Thakral S, Ghoshal K (2015) miR-122 is a unique molecule with great potentia in diagnosis, prognosis of liver disease, and therapy both as miRNA mimic and antimir. Curr Gene Ther 15: 142-150.

83. Hass HG, Nehls O, Jobst J, Frilling A, Vogel U, et al. (2008) Identification of osteopontin as the most consistently over-expressed gene in intrahepatic cholangiocarcinoma: detection by oligonucleotide microarray and real-time PCR analysis. World J Gastroenterol 14: 2501-2510.

84. Johnston NI, El-Tanani MK (2008) Osteopontin: A new role for a familiar actor Breast Cancer Res 10: 306

85. Zhang H, Ye QH, Ren N, Zhao L, Wang YF, et al. (2006) The prognostic significance of preoperative plasma levels of osteopontin in patients with hepatocellular carcinoma. J Cancer Res Clin Oncol 132: 709-717.

86. Zhang H, Liu HB, Yuan DM, Wang ZF, Wang YF, et al. (2014) Prognostic value of secreted phosphoprotein-1 in pleural effusion associated with non-small cell lung cancer. BMC Cancer 14: 280.

87. Huang CF, Yu GT, Wang WM, Liu B, Sun ZJ (2014) Prognostic and predictive values of SPP1, PAI and caveolin-1 in patients with oral squamous cell carcinoma. Int J Clin Exp Pathol 7: 6032-6039.

88. Rohde F, Rimkus C, Friederichs J, Rosenberg R, Marthen C, et al. (2007) Expression of osteopontin, a target gene of de-regulated Wht signaling, predicts survival in colon cancer. Int J Cancer 121: 1717-1723.

89. Cao DX, Li ZJ, Jiang XO, Lum YL, Khin E, et al. (2012) Osteopontin as potential biomarker and therapeutic target in gastric and liver cancers. World $\mathrm{J}$ Gastroenterol 18: 3923-3930. 
Citation: Severino FE, Hasimoto CN, Rodrigues MAM, Llanos JC, Reis PP (2017) Integrative Analysis of Transcriptome and MicroRNome Profiles in Cholangiocarcinoma. Cancer Sci Ther 9: 580-588. doi: 10.4172/1948-5956.1000477

90. Gardner HA, Berse B, Senger DR (1994) Specific reduction in osteopontin synthesis by antisense RNA inhibits the tumorigenicity of transformed Rat 1 fibroblasts. Oncogene 9: 2321-2326.

91. Yokosaki Y, Matsuura N, Sasaki T, Murakami I, Schneider H, et al. (1999) The integrin alpha(9)beta(1) binds to a novel recognition sequence (SVVYGLR) in the thrombin-cleaved amino-terminal fragment of osteopontin. J Biol Chem 274: 36328-36334.

92. Rangaswami H, Bulbule A, Kundu GC (2006) Osteopontin: Role in cell signaling and cancer progression. Trends Cell Biol 16: 79-87.

93. Fingleton B (2006) Matrix metalloproteinases: Roles in cancer and metastasis. Front Biosci 11: 479-491.

94. Sun Q, Zhao C, Xia L, He Z, Lu Z, et al. (2014) High expression of matrix metalloproteinase- 9 indicates poor prognosis in human hilar cholangiocarcinoma. Int J Clin Exp Pathol 7: 6157-6164.

95. Gialeli C, Theocharis AD, Karamanos NK (2011) Roles of matrix metalloproteinases in cancer progression and their pharmacological targeting. Febs J 278: 16-27.

96. Deng H, Guo RF, Li WM, Zhao M, Lu YY (2005) Matrix metalloproteinase 11 depletion inhibits cell proliferation in gastric cancer cells. Biochem Biophys Res Commun 326: 274-281.

97. Motrescu ER, Rio MC (2008) Cancer cells, adipocytes and matrix metalloproteinase 11: A vicious tumor progression cycle. Biol Chem 389: $1037-$ 1041.

98. Lichtinghagen R, Helmbrecht T, Arndt B, Böker KH (1995) Expression pattern of matrix metalloproteinases in human liver. Eur $\mathrm{J}$ Clin Chem Clin Biochem 33: $65-71$

99. Sternlicht MD, Werb Z (2001) How matrix metalloproteinases regulate cell behavior. Annu Rev Cell Dev Biol 17: 463-516.

100. Huang QX, Cui JY, Ma H (2016) Screening of potential biomarkers for cholangiocarcinoma by integrated analysis of microarray data sets. Cancer Gene Ther 23: 48-53.

101. Liu H, Zhang T, Li X, Huang J, Wu B, et al. (2008) Predictive value of MMP-7 expression for response to chemotherapy and survival in patients with nonsmall cell lung cancer. Cancer Sci 99: 2185-2192.

102. Urribarri AD, Munoz-Garrido $P$, Perugorria MJ, Erice O, Merino-Azpitarte M, et al. (2014) Inhibition of metalloprotease hyperactivity in cystic cholangiocytes halts the development of polycystic liver diseases. Gut 63: 1658-1667. 